\title{
Replacement of Ensemble Averaging by the Use of a Broadband Source in Scattering of Light from a One-Dimensional Randomly Rough Interface between Two Dielectric Media
}

\author{
Alexei A. Maradudin ${ }^{1}$ and Ingve Simonsen $\mathbb{D D}^{2,3}$ \\ ${ }^{1}$ Department of Physics and Astronomy, University of California, Irvine, CA 92697, USA \\ ${ }^{2}$ Surface du Verre et Interfaces, UMR 125 CNRS/Saint-Gobain, 93303 Aubervilliers, France \\ ${ }^{3}$ Department of Physics, NTNU-Norwegian University of Science and Technology, NO-7491 Trondheim, Norway
}

Correspondence should be addressed to Ingve Simonsen; ingve.simonsen@ntnu.no

Received 11 July 2017; Accepted 8 October 2017; Published 21 January 2018

Academic Editor: Pasquale Imperatore

Copyright (c) 2018 Alexei A. Maradudin and Ingve Simonsen. This is an open access article distributed under the Creative Commons Attribution License, which permits unrestricted use, distribution, and reproduction in any medium, provided the original work is properly cited.

By the use of phase perturbation theory we show that if a single realization of a one-dimensional randomly rough interface between two dielectric media is illuminated at normal incidence from either medium by a broadband Gaussian beam, it produces a scattered field whose differential reflection coefficient closely matches the result produced by averaging the differential reflection coefficient produced by a monochromatic incident beam over the ensemble of realizations of the interface profile function.

\section{Introduction}

In theoretical calculations of some property of monochromatic light scattered from, or transmitted through, a randomly rough surface, such as the angular or spatial dependence of its intensity, what is actually calculated is the average of that property over the ensemble of realizations of the random surface profile. This procedure averages over the speckles that would be produced if monochromatic light were scattered by or transmitted through a single realization of the random surface and produces a smooth angular or spatial dependence of the property of interest.

In an experiment, this property is measured for a single realization of the random surface. If the surface is illuminated by a monochromatic source, the resulting speckles have to be averaged in some way to produce the kind of smooth curve that ensemble averaging yields. This can be done by rotating or dithering the sample. However, in some cases, moving the surface is not an option. In such cases, one can exploit the fact that the speckle pattern depends on the wavelength of the incident light [1] to average over the speckles by using a broadband (polychromatic) beam to illuminate the surface instead of a monochromatic one. In an earlier paper [2], it was demonstrated that illuminating one realization of a one-dimensional randomly rough perfectly conducting surface by an s-polarized broadband Gaussian beam produced an intensity profile of the scattered field that closely matched the one produced by averaging the intensity of the scattered field produced by a monochromatic incident beam over the ensemble of realizations of the random surface profile function.

In this paper, we explore the replacement of ensemble averaging by the use of an incident broadband Gaussian beam in the more realistic case where the one-dimensional rough interface between two dielectric media is illuminated at normal incidence from either medium and the differential reflection coefficient of the scattered light is sought.

\section{Scattering Theory}

The system we study consists of a dielectric medium whose dielectric constant is $\varepsilon_{1}$ in the region $x_{3}>\zeta\left(x_{1}\right)$ and a dielectric medium whose dielectric constant is $\varepsilon_{2}$ in the region $x_{3}<\zeta\left(x_{1}\right)$ (Figure 1). Both $\varepsilon_{1}$ and $\varepsilon_{2}$ are assumed to be real, positive, and frequency independent. The interface profile function $\zeta\left(x_{1}\right)$ is assumed to be a single-valued function of 


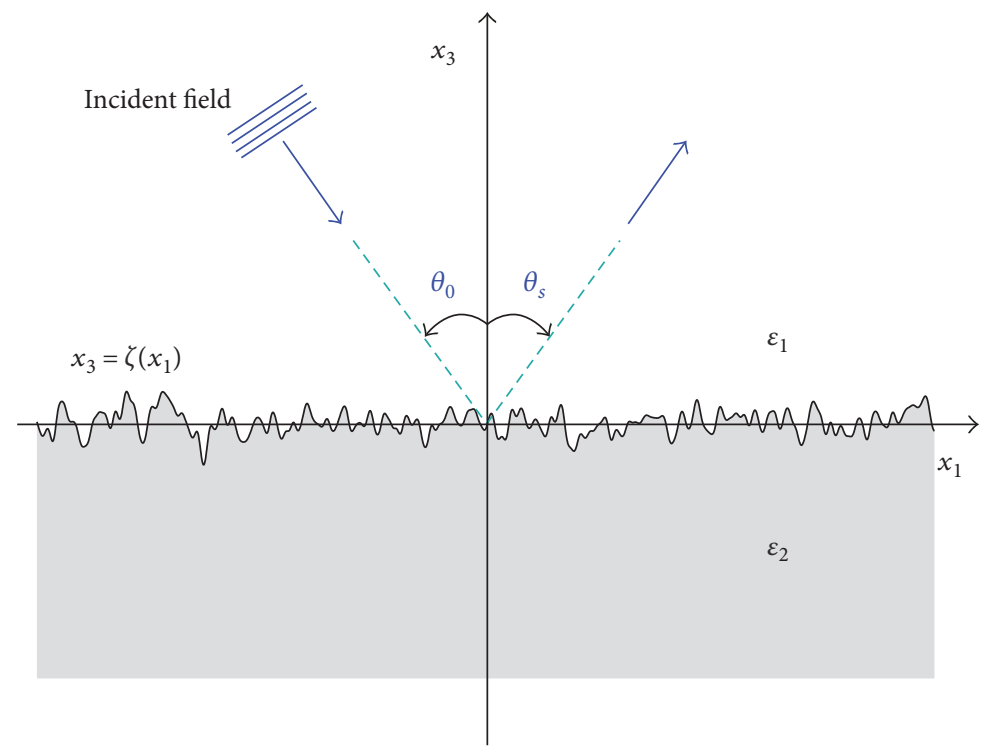

FIGURE 1: Schematics of the scattering geometry.

$x_{1}$ that is differentiable and constitutes a random process. This interface is illuminated at normal incidence from the region $x_{3}>\zeta\left(x_{1}\right)$ by a $\mathrm{p}$ - or s-polarized broadband Gaussian beam of light of angular frequency $\omega$, whose plane of incidence is the $x_{1} x_{3}$ plane. The single nonzero component of its electromagnetic field is a weighted superposition of incoming plane waves,

$$
\begin{aligned}
& F_{v}\left(x_{1}, x_{3} ; t\right)_{\text {inc }} \\
& \quad=\int_{-\infty}^{\infty} \frac{\mathrm{d} \omega}{2 \pi} \int_{-\sqrt{\varepsilon_{1} \frac{\omega}{c}}}^{\sqrt{\varepsilon_{1} \frac{\omega}{c}}} \frac{\mathrm{d} k}{2 \pi} W(k, \omega) \exp \left[\mathrm{i} k x_{1}-\mathrm{i} \alpha_{1}(k, \omega) x_{3}-\mathrm{i} \omega t\right],
\end{aligned}
$$

where $F_{v}\left(x_{1}, x_{3} ; t\right)_{\text {inc }}$ is $H_{2}\left(x_{1}, x_{3} ; t\right)_{\text {inc }}$ when $v=p$ and is $E_{2}\left(x_{1}, x_{3} ; t\right)$ when $v=s$. The function $\alpha_{1}(k, \omega)$ is defined by $\alpha_{1}(k, \omega)=\left[\varepsilon_{1}(\omega / c)^{2}-k^{2}\right]^{1 / 2}$ with $\operatorname{Re} \alpha_{1}(k, \omega)>0$ and $\operatorname{Im} \alpha_{1}(k, \omega)>0$, where $c$ is the speed of light in vacuum. The weight function $W(k, \omega)$ has the factored form

$$
W(k, \omega)=G(k) F(\omega)
$$

where

$G(k)=\frac{2 \sqrt{\pi}}{\alpha_{1}(k, \omega)} \sqrt{\varepsilon_{1}} \frac{w \omega}{2 c} \exp \left[-\left(\sqrt{\varepsilon_{1}} \frac{w \omega}{2 c}\right)^{2} \arcsin ^{2}\left(\frac{k c}{\sqrt{\varepsilon_{1}} \omega}\right)\right]$,

while $F(\omega)$ is a random function that possesses the properties

$$
\begin{aligned}
& \left\langle F(\omega) F^{*}\left(\omega^{\prime}\right)\right\rangle_{F}=2 \pi \delta\left(\omega-\omega^{\prime}\right) S_{0}(\omega), \\
& \left\langle F(\omega) F\left(\omega^{\prime}\right)\right\rangle_{F}=0 .
\end{aligned}
$$

The angle brackets $\langle\cdots\rangle_{F}$ here denote an average over the ensemble of realizations of the field [3]. An incident field of this nature is produced by a superluminescent diode [4], for example. We assume that the spectral density of the incident field $S_{0}(\omega)$ has a Gaussian form with a central frequency $\omega_{0}$ and a $1 / e$ halfwidth $\Delta \omega$,

$$
S_{0}(\omega)=\frac{1}{\sqrt{\pi} \Delta \omega} \exp \left[-\left(\frac{\omega-\omega_{0}}{\Delta \omega}\right)^{2}\right]
$$

In the following, it will be assumed that the halfwidth is small enough that the spectral density of the incident light can be regarded as zero when $\omega<0$. Moreover, for convenience, the function $F(\omega)$ will be regarded as zero when $\omega<0$.

In the limit that $\sqrt{\varepsilon_{1}} w \omega / 2 c \gg 1$, that will be assumed here, (1) represents a Gaussian beam of 1/e halfwidth $w$ that is incident normally on the rough interface. To see this, one will make the change of variable $k=\sqrt{\varepsilon_{1}}(\omega / c) \sin \theta$ in (1) which results in a Gaussian integral on the right-hand side of this equation that is evaluated analytically to produce

$F_{v}\left(x_{1}, x_{3} ; t\right)_{\text {inc }}=\int_{-\infty}^{\infty} \frac{\mathrm{d} \omega}{2 \pi} F(\omega) \exp \left[-\left(\frac{x_{1}}{w}\right)^{2}-\mathrm{i} \sqrt{\varepsilon_{1}} \frac{\omega}{c} x_{3}-\mathrm{i} \omega t\right]$.

Due to the linearity of the scattering problem, the scattered field can be written as

$$
\begin{aligned}
F_{v}\left(x_{1}, x_{3} ; t\right)_{s c}= & \int_{-\infty}^{\infty} \frac{\mathrm{d} \omega}{2 \pi} F(\omega) \int_{-\sqrt{\varepsilon_{1} \frac{\omega}{c}}}^{\sqrt{\varepsilon_{1} \frac{\omega}{c}}} \frac{\mathrm{d} k}{2 \pi} G(k) \\
& \cdot \int_{-\infty}^{\infty} \frac{\mathrm{d} q}{2 \pi} R_{v}(q \mid k) \exp \left[\mathrm{i} q x_{1}+\mathrm{i} \alpha_{1}(q, \omega) x_{3}-\mathrm{i} \omega t\right],
\end{aligned}
$$

where $R_{v}(q \mid k)$ is the scattering amplitude that is obtained when the incident field is given by $\exp \left[\mathrm{i} k x_{1}-\mathrm{i} \alpha_{1}(k, \omega) x_{3}-\mathrm{i} \omega t\right]$ or 


$$
\begin{aligned}
& F_{v}\left(r, \theta_{s} ; t\right)_{\mathrm{sc}} \\
& =\int_{-\infty}^{\infty} \frac{\mathrm{d} \omega}{2 \pi} F(\omega) \int_{-\sqrt{\varepsilon_{1}} \frac{\omega}{c}}^{\sqrt{\varepsilon_{1}} \frac{\omega}{c}} \frac{\mathrm{d} k}{2 \pi} G(k) \\
& \quad \cdot \int_{-\infty}^{\infty} \frac{\mathrm{d} q}{2 \pi} R_{v}(q \mid k) \exp \left[\mathrm{i} q r \sin \theta_{s}+\mathrm{i} a_{1}(q, \omega) r \cos \theta_{s}-\mathrm{i} \omega t\right],
\end{aligned}
$$

where $r=\sqrt{x_{1}^{2}+x_{3}^{2}}$ and $x_{1}=r \sin \theta_{s}, x_{3}=r \cos \theta_{s}$. It can be calculated by any of the several approaches such as small amplitude perturbation theory [5], the Kirchhoff approximation [6], phase-perturbation theory [7], and by rigorous numerical solutions of the equations of scattering theory [8]. We will use here the first-order phase perturbation theory expression for $R_{v}(q \mid k)$, due to its simplicity and because it interpolates between small-amplitude perturbation theory and the Kirchhoff approximation.

In small-amplitude perturbation theory, the expression obtained for $R_{v}(q \mid k)$ to the lowest nonzero order in the interface profile function $\zeta\left(x_{1}\right)$ is

$$
R_{v}(q \mid k)=R_{v}(k)\left[2 \pi \delta(q-k)+\mathrm{i} \Phi_{v}(q \mid k) \widehat{\zeta}(q-k)+\cdots\right],
$$

where

$$
\widehat{\zeta}(Q)=\int_{-\infty}^{\infty} \mathrm{d} x_{1} \zeta\left(x_{1}\right) \exp \left(-\mathrm{i} Q x_{1}\right)
$$

while

$$
\begin{aligned}
R_{p}(k)= & \frac{\varepsilon_{2} \alpha_{1}(k, \omega)-\varepsilon_{1} \alpha_{2}(k, \omega)}{\varepsilon_{2} \alpha_{1}(k, \omega)+\varepsilon_{1} \alpha_{2}(k, \omega)}, \\
\Phi_{p}(q \mid k)= & \frac{\varepsilon_{2}-\varepsilon_{1}}{\varepsilon_{2} \alpha_{1}(q, \omega)+\varepsilon_{1} \alpha_{2}(q, \omega)}\left[\varepsilon_{2} q k-\varepsilon_{1} \alpha_{2}(q, \omega) \alpha_{2}(k, \omega)\right] \\
& \cdot \frac{2 \alpha_{1}(k, \omega)}{\varepsilon_{2} a_{1}(k, \omega)-\varepsilon_{1} \alpha_{2}(k, \omega)},
\end{aligned}
$$

and

$$
\begin{aligned}
R_{s}(k) & =\frac{\alpha_{1}(k, \omega)-\alpha_{2}(k, \omega)}{\alpha_{1}(k, \omega)+\alpha_{2}(k, \omega)}, \\
\Phi_{s}(q \mid k) & =\left[\alpha_{2}(q, \omega)-\alpha_{1}(q, \omega)\right] \frac{2 \alpha_{1}(k, \omega)}{\alpha_{1}(k, \omega)-\alpha_{2}(k, \omega)},
\end{aligned}
$$

with $\alpha_{2}(k, \omega)=\left[\varepsilon_{2}(\omega / c)^{2}-k^{2}\right]^{1 / 2}, \operatorname{Re} \alpha_{2}(k, \omega)>0$, and $\operatorname{Im} \alpha_{2}$ $(k, \omega)>0$. We can rewrite the right-hand side of (9) as a Fourier integral,

$$
\begin{aligned}
& R_{v}(q \mid k) \\
& \quad=R_{v}(k) \int_{-\infty}^{\infty} \mathrm{d} x_{1} \exp \left[-\mathrm{i}(q-k) x_{1}\right]\left[1+\mathrm{i} \Phi_{v}(q \mid k) \zeta\left(x_{1}\right)+\cdots\right] .
\end{aligned}
$$

On exponentiating the expression in brackets in the integrand in this expression, we obtain the first-order phase perturbation theory expression for $R_{v}(q \mid k)$,

$$
R_{v}(q \mid k)=R_{v}(k) \int_{-\infty}^{\infty} \mathrm{d} x_{1} \exp \left[-\mathrm{i}(q-k) x_{1}\right] \exp \left[\mathrm{i} \Phi_{v}(q \mid k) \zeta\left(x_{1}\right)\right]
$$

We will use this expression here due to its simplicity. After interchanging the order of the $k$ and $q$ integrations in (7), it follows that the scattering amplitude for a Gaussian monochromatic beam of frequency $\omega$ can be expressed as

$$
R_{v}(q, \omega)=\int_{-\sqrt{\varepsilon_{1}} \frac{\omega}{c}}^{\sqrt{\varepsilon_{1} \frac{\omega}{c}}} \frac{\mathrm{d} k}{2 \pi} R_{v}(q \mid k) G(k),
$$

so that the scattered field becomes

$$
\begin{aligned}
F_{v}\left(x_{1}, x_{3} ; t\right)_{\mathrm{sc}}= & \int_{-\infty}^{\infty} \frac{\mathrm{d} \omega}{2 \pi} F(\omega) \\
& \cdot \int_{-\infty}^{\infty} \frac{\mathrm{d} q}{2 \pi} R_{v}(q, \omega) \exp \left[\mathrm{i} q x_{1}+\mathrm{i} \alpha_{1}(q, \omega) x_{3}-\mathrm{i} \omega t\right]
\end{aligned}
$$

The scattering amplitude $R_{v}(q, \omega)$ enters the definition of the differential reflection coefficient which is defined as the fraction of the power flux incident onto the rough interface that is scattered into an angular interval of width $d \theta_{s}$ about the scattering angle $\theta_{s}$ [9]. For an illumination of the random interface by a normally incident Gaussian beam of frequency $\omega$, the expression for the differential reflection coefficient in the wide beam limit $\sqrt{\varepsilon_{1}} w \omega / 2 c \gg 1$ reads $[8,10]$

$$
\frac{\partial R_{v}}{\partial \theta_{s}}\left(\theta_{s}\right)=\frac{\sqrt{\varepsilon_{1}}}{\sqrt{2} \pi^{3 / 2}} \frac{\omega}{c w} \cos ^{2} \theta_{s}|R(q, \omega)|^{2},
$$

where $q=\sqrt{\varepsilon_{1}}(\omega / c) \sin \theta_{s}$. When the differential reflection coefficient from (17), for monochromatic illumination at frequency $\omega_{0}$, is averaged over an ensemble of realizations of the random interface, the mean differential reflection coefficient is obtained and we denote it $\left\langle\partial R_{v} / \partial \theta_{s}\right\rangle$ in the following. On the other hand, if the illumination of the random interface is done by a broadband source and characterized by the center frequency $\omega_{0}$ and the halfwidth is $\Delta \omega$, the "broadband" differential reflection coefficient $\left\langle\partial R_{v} / \partial \theta_{s}\right\rangle_{F}$ is obtained.

We now turn to the calculation of a simple expression for the scattering amplitude $R_{v}(q, \omega)$. On substituting into (15), the results from (14) and (3) and making the change of variable $k=\sqrt{\varepsilon_{1}}(\omega / c) \sin \theta$ in the resulting expression, one gets

$$
\begin{aligned}
R_{v}(q, \omega)= & \frac{\sqrt{\varepsilon_{1}}}{\pi} \frac{w \omega}{2 c} \int_{-\pi / 2}^{\pi / 2} \mathrm{~d} \theta \exp \left[-\left(\sqrt{\varepsilon_{1}} \frac{w \omega}{2 c}\right)^{2} \theta^{2}\right] R_{v}\left(\sqrt{\varepsilon_{1}} \frac{\omega}{c} \sin \theta\right) \\
& \times \int_{-\infty}^{\infty} \mathrm{d} x_{1} \exp \left[-\mathrm{i} q x_{1}+\mathrm{i} \sqrt{\varepsilon_{1}} \frac{\omega}{c} \sin \theta x_{1}\right] \\
& \exp \left[\mathrm{i} \Phi_{v}\left(q \mid \sqrt{\varepsilon_{1}} \frac{\omega}{c} \sin \theta\right) \zeta\left(x_{1}\right)\right] .
\end{aligned}
$$

On passing to the limit $\sqrt{\varepsilon_{1}}(w \omega / 2 c) \gg 1$, corresponding to a wide Gaussian beam, one may take advantage of the 
approximations $\sin \theta \approx 0$ and $\cos \theta \approx 1$, with the consequence that $\Phi_{v}$ becomes a linear function of the variable $\theta$,

$$
\Phi_{v}\left(q \mid \sqrt{\varepsilon_{1}} \frac{\omega}{c} \sin \theta\right) \approx \phi_{v}^{(0)}(q, \omega)+\phi_{v}^{(1)}(q, \omega) \theta
$$

with

$$
\phi_{v}^{(0)}(q, \omega)=\Phi_{v}(q \mid 0)
$$

and

$$
\begin{aligned}
& \phi_{p}^{(1)}(q, \omega)=2 \frac{\omega}{c} \sqrt{\varepsilon_{1} \varepsilon_{2}}\left(\sqrt{\varepsilon_{1}}+\sqrt{\varepsilon_{2}}\right) \frac{q}{\varepsilon_{2} \alpha_{1}(q, \omega)+\varepsilon_{1} \alpha_{2}(q, \omega)} \\
& \phi_{s}^{(1)}(q, \omega)=0 .
\end{aligned}
$$

Moreover, in the limit $\sqrt{\varepsilon_{1}}(w \omega / 2 c) \gg 1$, the $\theta$ integral in (18) takes the Gaussian form, due to the results in (19)-(21) and can hence be evaluated analytically with the result that

$$
\begin{aligned}
R_{v}(q, \omega)=R_{v}(0) \int_{-\infty}^{\infty} \mathrm{d} x_{1} \exp [ & -\left\{\frac{x_{1}}{w}+\frac{\phi_{v}^{(1)}(q, \omega) \zeta\left(x_{1}\right)}{\sqrt{\varepsilon_{1}}(\omega / c) w}\right\}^{2} \\
& \left.-\mathrm{i} q x_{1}+\mathrm{i} \phi_{v}^{(0)}(q, \omega) \zeta\left(x_{1}\right)\right]
\end{aligned}
$$

This is the simplified expression for the scattering amplitude derived from phase perturbation theory that we will use to produce the results to be presented later in this paper. It represents a significant simplification relative to, for instance, obtaining the scattering amplitude by rigorous means [8], which requires solving a linear system of equations that becomes time-consuming when the interface becomes long.

The interface profile function $\zeta\left(x_{1}\right)$ is assumed to be a single-valued function of $x_{1}$ that is differentiable and constitutes a zero-mean, stationary Gaussian random process defined by

$$
\left\langle\zeta\left(x_{1}\right) \zeta\left(x_{1}^{\prime}\right)\right\rangle=\delta^{2} W\left(\left|x_{1}-x_{1}^{\prime}\right|\right)
$$

The angle brackets here denote an average over the ensemble of realizations of $\zeta\left(x_{1}\right), \delta=\left\langle\zeta^{2}\left(x_{1}\right)\right\rangle^{1 / 2}$ is the root meansquare height of the interface, and $W\left(\left|x_{1}\right|\right)$ is the normalized interface height auto-correlation function.

The power spectrum of the interface roughness, $g(|k|)$, is the Fourier transform of $W\left(\left|x_{1}\right|\right)$,

$$
g(|k|)=\int_{-\infty}^{\infty} \mathrm{d} x_{1} W\left(\left|x_{1}\right|\right) \exp \left(-\mathrm{i} k x_{1}\right)
$$

In the calculations carried out in this work, $W\left(\left|x_{1}\right|\right)$, will be assumed to have the Gaussian form

$$
W\left(\left|x_{1}\right|\right)=\exp \left(-\frac{x_{1}^{2}}{a^{2}}\right)
$$

where the characteristic length $a$ is the transverse correlation length if the interface roughness. The power spectrum $g(|k|)$ in this case also has the Gaussian form

$$
g(|k|)=\sqrt{\pi} a \exp \left(-\frac{k^{2} a^{2}}{4}\right)
$$

A single realization of the interface profile function is given by [11]

$$
\begin{aligned}
\zeta\left(x_{1}\right)= & \delta \sqrt{\frac{2}{\mathscr{L}}} \sum_{m=1}^{\infty}\left[g\left(\frac{2 \pi m}{L}\right)\right]^{1 / 2} \\
& \cdot\left[\xi_{2 m-1} \sin \left(\frac{2 \pi m x_{1}}{\mathscr{L}}\right)+\xi_{2 m} \cos \left(\frac{2 \pi m x_{1}}{\mathscr{L}}\right)\right] .
\end{aligned}
$$

In this expression, the $\left\{\xi_{m}\right\}$ are independent Gaussian random deviates with zero mean and unit variance:

$$
\left\langle\xi_{m}\right\rangle=0, \quad\left\langle\xi_{m}^{2}\right\rangle=1
$$

The function defined by (27) is a periodic function of $x_{1}$ with a period $\mathscr{L}$. To avoid edge effects, only the portion of this function in the interval $-L / 2<x_{1}<L / 2$ where $L=\mathscr{L} / 2$ is used in calculations.

\section{Results and Discussion}

In Figure 2(a), we present a plot of the speckle pattern of the scattered field as a function of the scattering angle $\theta_{s}$ produced by a monochromatic p-polarized Gaussian beam of frequency $\omega_{0}$, given by (6) with $F(\omega)=2 \pi \delta\left(\omega-\omega_{0}\right)$, incident on a single realization of a one-dimensional randomly rough interface generated with the use of (27). In Figure 2(b), we present the differential reflection coefficient of the scattered field given by (16) when the realization of the interface profile function used in obtaining Figure 2(a) is illuminated by a broadband Gaussian beam whose center frequency is $\omega_{0}$ with a halfwidth $\Delta \omega=0.2 \omega_{0}$. To obtain this result, calculations of the differential reflection coefficient were carried out for several values of $\Delta \omega$, ranging from $0.2 \omega_{0}$ to $0.4 \omega_{0}$. The results did not differ in any significant way so we chose to use the smallest of these $\Delta \omega$ values. It should be remarked that when a different realization of the surface was illuminated by the same broadband beam used to produce the result in Figure 2(b), the result was essentially $\left\langle\partial R_{p} / \partial \theta_{s}\right\rangle_{F}$ from the same figure except for some small-amplitude fine details. Finally, in Figure 2(c), we plot the mean differential reflection coefficient $\left\langle\partial R_{p} / \partial \theta_{s}\right\rangle$ obtained by averaging the results from $N_{p}=10,000$ realizations of the interface profile function generated by (27) when the interface is illuminated by the same monochromatic Gaussian beam of frequency $\omega_{0}$ used in obtaining Figure 2(a). The values of the theoretical 


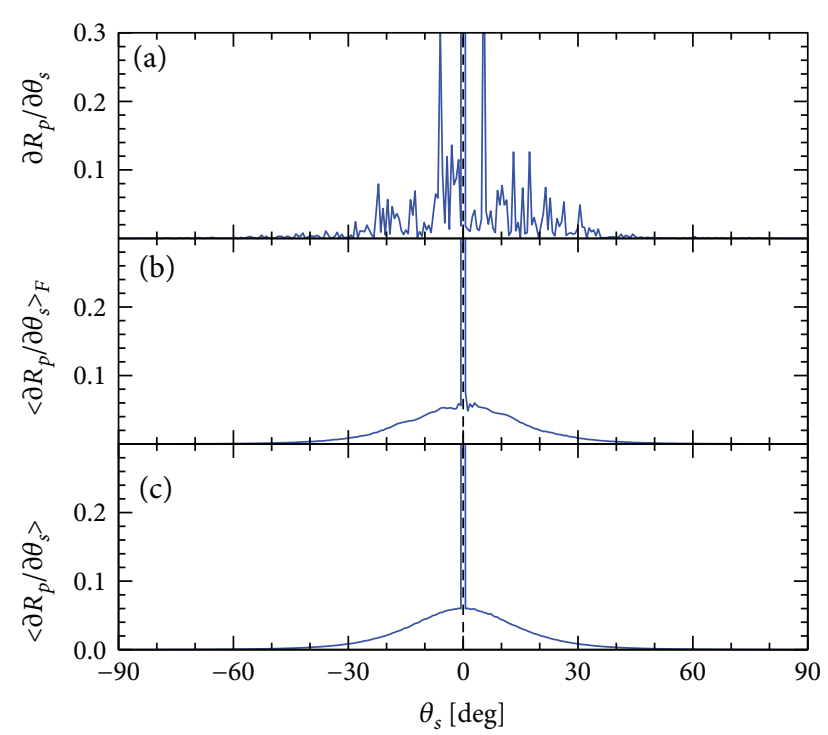

Figure 2: The differential reflection coefficient $\partial R_{p} / \partial \theta_{s}$ defined by (17) for a vacuum-glass system $\left[\varepsilon_{1}=1\right.$ and $\varepsilon_{2}=2$.25] illuminated from the vacuum side by different p-polarized incident beams: (a) a Gaussian monochromatic beam of halfwidth $w$ and frequency $\omega_{0}$; (b) a broadband Gaussian beam of center frequency $\omega_{0}$ and frequency bandwidth $\Delta \omega=0.2 \omega_{0}$; and (c) a monochromatic beam as in Figure 2(a) but with an average performed over an ensemble of $N_{p}=10,000$ realizations of the interface profile function. The angle of incidence was $\theta_{0}=0^{\circ}$ and the wavelength was $\lambda_{0}=2 \pi \mathrm{cl}$ $\omega_{0}=632.8 \mathrm{~nm}$. The randomly rough interface was characterized by the parameters $\delta=0.15 \lambda_{0}, a=1.50 \lambda_{0}$, and $L=10^{4} \lambda_{0}$, and the sampling interval used to discretize the surface was $\Delta x_{1}=\lambda_{0} / 10$. For the width of the Gaussian beam, the value $w=L / 4$ was used. The same random interface was used in producing the results of the first two panels of this figure.

and experimental parameters assumed in obtaining these results were $\varepsilon_{1}=1, \varepsilon_{2}=2.25, \lambda_{0}=2 \pi c / \omega_{0}=632.8 \mathrm{~nm}$, and $w=L / 4$ where $L$ is the length of the surface. The parameters defining the interface roughness were $\delta=0.15 \lambda_{0}, a=1.50 \lambda_{0}$, and $L=10^{4} \lambda_{0}$. The sampling interval used was $\Delta x_{1}=\lambda_{0} / 10$ so that the interface was discretized onto $N=10^{5}$ points. Figure 3 presents corresponding results for s-polarized incident beams.

The results presented in Figures 2 and 3 show that the use of a broadband beam in illuminating a single realization of a one-dimensional randomly rough interface averages over the speckles produced by a monochromatic beam. It therefore produces a differential reflection coefficient that closely matches the one produced by a monochromatic beam when the resulting differential reflection coefficient is averaged over the ensemble of realizations of the interface profile function.

To facilitate the comparison of $\left\langle\partial R_{v} / \partial \theta_{s}\right\rangle_{F}$ and $\left\langle\partial R_{v} / \partial \theta_{s}\right\rangle$, in Figures 4(a) and 4(c), we plot simultaneously these quantities on a semilogarithmic scale. It is observed that the agreement between them is rather good even in the tails of the scattered intensity distributions.

The results presented in Figures 2 and 3 were, for convenience, all obtained under the assumption of phase

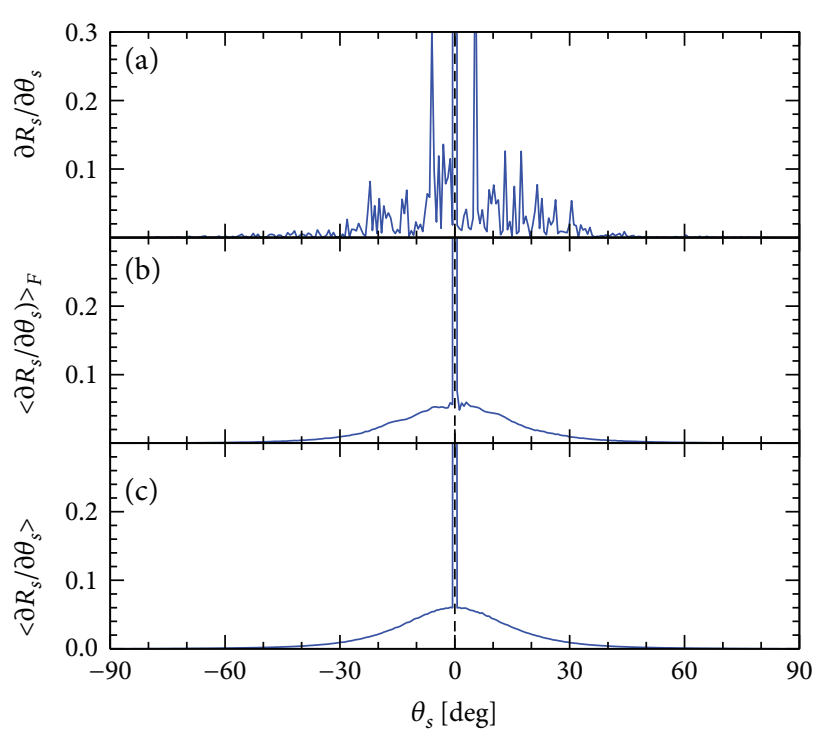

Figure 3: Same as Figure 2 but for s-polarized incident beams, the single realization of the rough interface used to obtain the results of the first two panels of this figure is the same one used in Figure 2.

perturbation theory. In Figure 4, we compare the mean differential reflection coefficients $\left\langle\partial R_{v} / \partial \theta_{s}\right\rangle$ from Figures 2(c) and $3(\mathrm{c})$ to rigorous computer simulation results obtained by solving the equations of scattering theory [8]. The agreement between the two sets of results is rather convincing. In passing, it should be noted that in performing the rigorous simulations the length used for the rough interface was $L^{\prime}=10^{2} \lambda_{0}$ and the width of the Gaussian beam was $L^{\prime} / 4$. This length of the rough interface is two orders of magnitude shorter than the length used in obtaining the results based on phase perturbation theory.

We now turn to a scattering geometry where the medium of incidence is the optically denser medium. Here, we assume a glass-vacuum system characterized by $\varepsilon_{1}=$ 2.25 and $\varepsilon_{2}=1$. Physically, this corresponds to the light being incident from the opposite side of the rough interface relative to the system we previously studied. The results for the differential reflection coefficients for the glass-vacuum system are presented in Figures 5, 6, and 7. In these figures, the angles for which $\left|\theta_{s}\right| \geq \theta_{s}^{*}$ have been indicated as shaded regions, where $\theta_{s}^{*}=\arcsin \left(\sqrt{\varepsilon_{2} / \varepsilon_{1}}\right)=41.81^{\circ}$ denotes the critical angle for total internal reflection. Due to the assumptions underlying phase perturbation theory, it is expected not to work well when the angles of incidence and/or scattering in absolute value are larger than this critical angle. Hence, in Figures 5, 6, and 7, we have plotted the results obtained on the basis of phase perturbation theory only for $\left|\theta_{s}\right|<\theta_{s}^{*}$.

On the basis of the results presented in Figures 5, 6, and 7 , it is concluded that also for systems where the medium of incidence is the optically denser medium, one finds that the differential reflection coefficients $\left\langle\partial R_{v} / \partial \theta_{s}\right\rangle_{F}$ and $\left\langle\partial R_{v} / \partial \theta_{s}\right\rangle$ match each other rather well in the angular interval $\left|\theta_{s}\right|<\theta_{s}^{*}$. 


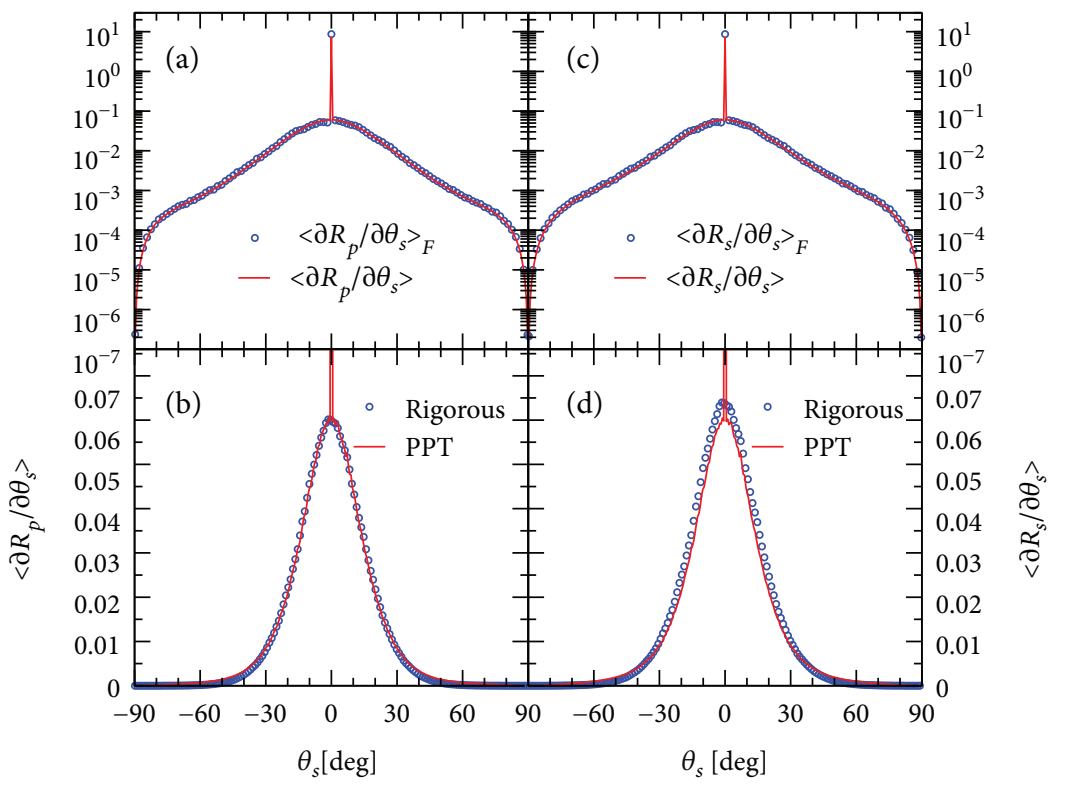

Figure 4: The quantities $\left\langle\partial R_{v} / \partial \theta_{s}\right\rangle_{F}$ and $\left\langle\partial R_{v} / \partial \theta_{s}\right\rangle$ obtained for a vacuum-glass system $\left[\varepsilon_{1}=1\right.$ and $\left.\varepsilon_{2}=2.25\right]$ plotted on semilogarithmic scales for $v=p$ [Figure 4(a)] and $v=s$ [Figure 4(c)]. Comparison of the mean differential reflection coefficients $\left\langle\partial R_{v} / \partial \theta_{s}\right\rangle$ obtained by either a rigorous simulation approach or on the basis of phase perturbation theory (PPT) for p-polarized (Figure 4(b)) or s-polarized (Figure 4(d)) illumination. All ensemble averaged quantities were obtained on the basis of $N_{p}=10,000$ realizations of the interface profile function. The remaining parameters are identical to those of Figure 2.

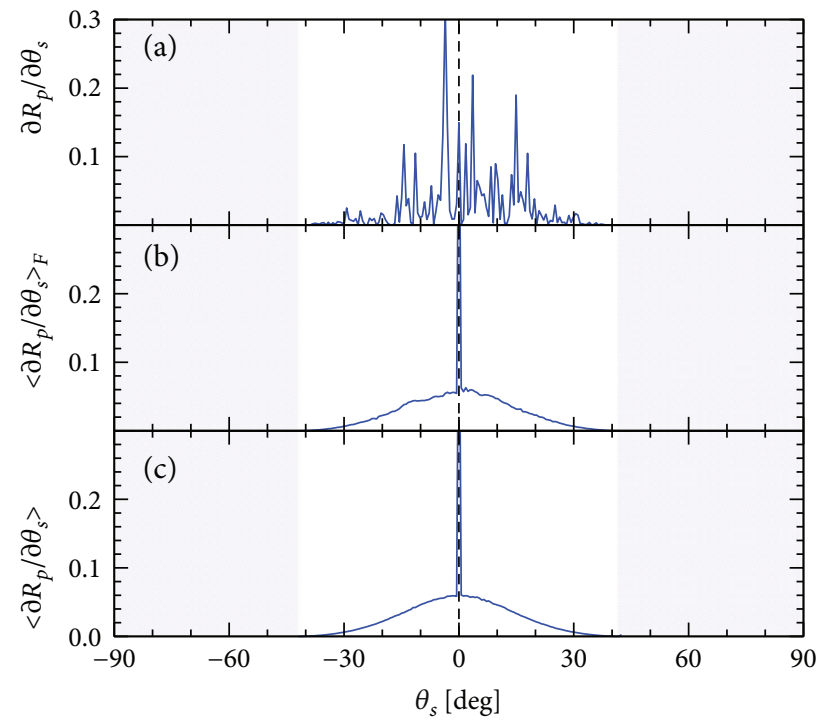

Figure 5: Same as Figure 2, that is, p-polarized illumination, but for a system where the medium of incidence is the optically denser medium $\left(\varepsilon_{1}=2.25\right.$ and $\left.\varepsilon_{2}=1\right)$, the shaded regions correspond to $\left|\theta_{s}\right| \geq \theta_{s}^{*}$ where $\theta_{s}^{*}=\arcsin \left(\sqrt{\varepsilon_{2} / \varepsilon_{1}}\right)=41.81^{\circ}$ denotes the critical angle for total internal reflection. The results are presented only for $\left|\theta_{s}\right|<\theta_{s}^{*}$.

\section{Conclusions}

The scattering of normally incident p- or s-polarized light from a one-dimensional randomly rough interface between two dielectric media is studied. Based on phase perturbation theory, it is demonstrated that using a broadband Gaussian

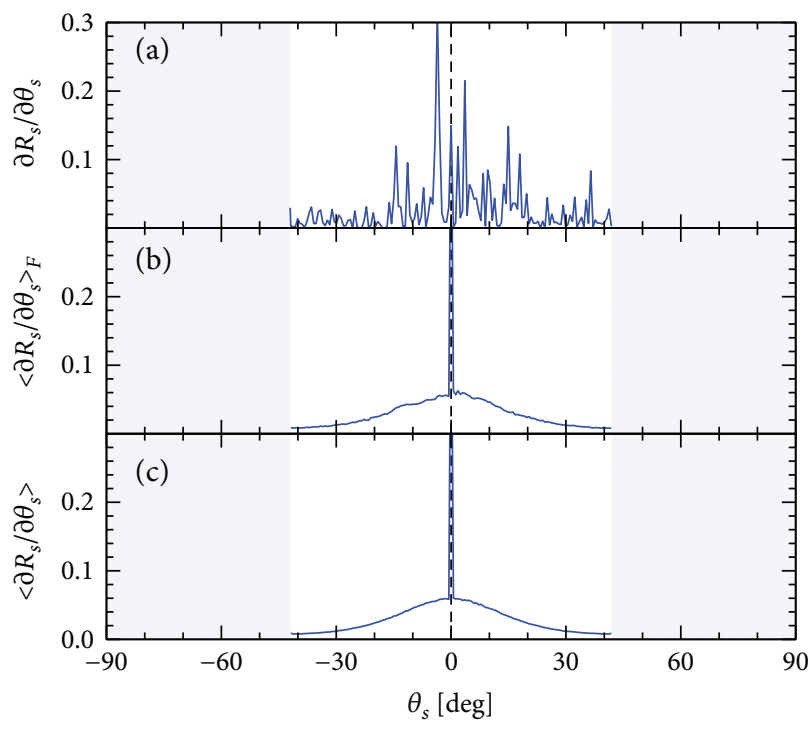

Figure 6: Same as Figure $5\left(\varepsilon_{1}=2.25\right.$ and $\left.\varepsilon_{2}=1\right)$ but assuming spolarized illumination.

beam to illuminate the surface produces a differential reflection coefficient that closely matches the one produced by a monochromatic Gaussian beam when the resulting differential reflection coefficient is averaged over the ensemble of realizations of the interface profile function. This result is obtained since the broadband beam averages over the speckles produced by a monochromatic beam.

The confirmation of the conjecture prompting the present work by these proof-of-concept calculations encourages 


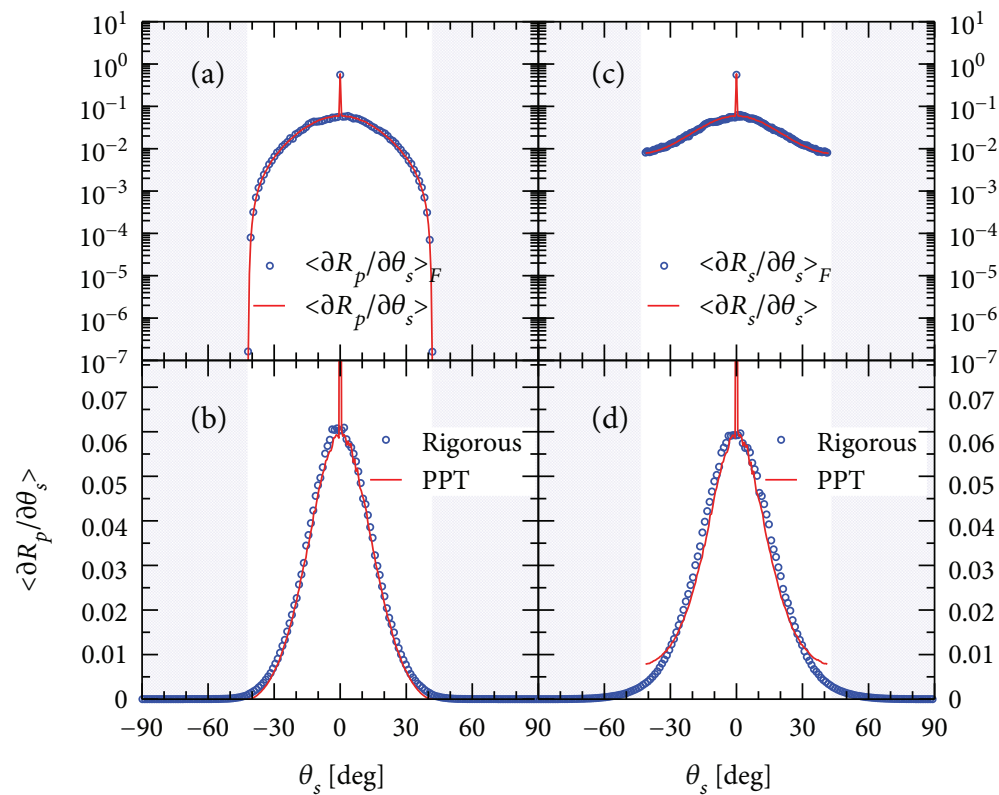

Figure 7: Same as Figure 4 but for a glass-vacuum system $\left(\varepsilon_{1}=2.25\right.$ and $\left.\varepsilon_{2}=1\right)$.

additional calculations using rigorous computer simulation methods instead of the phase perturbation theory approach, to explore the efficacy of a broadband source in calculations of rough surface scattering phenomena and in experimental studies of them.

\section{Conflicts of Interest}

The authors declare that there are no conflicts of interest regarding the publication of this paper.

\section{Acknowledgments}

The research of Ingve Simonsen was supported in part by the Research Council of Norway (contract 216699) and the French National Research Agency (ANR-15-CHIN-0003).

\section{References}

[1] J. W. Goodman, "Some fundamental properties of speckle," Journal of the Optical Society of America, vol. 66, no. 11, pp. 1145-1150, 1976.

[2] A. A. Maradudin, T. A. Leskova, and E. R. Méndez, "Replacement of ensemble averaging by the use of a broadband source in scattering from randomly rough surfaces," in 23rd Annual Review of Progress in Applied Computational Electromagnetics (ACES, University, MS), pp. 1915-1919, 2007.

[3] J. M. Stone, Radiation and Optics, McGraw-Hill, New York, 1963, Sec. 12-6.

[4] C. W. Tsai, Y. C. Chang, G. S. Shmavonyan, Y. S. Su, and C. F. Lin, "Extremely broadband superluminescent diodes/ semiconductor optical amplifiers in optical communications band," in Proc. SPIE 4989, Optical Devices for Fiber Communication IV, pp. 69-77, 2003.

[5] F. Toigo, A. Marvin, V. Celli, and N. R. Hill, "Optical properties of rough surfaces: general theory and the small roughness limit," Physical Review B, vol. 15, pp. 5618-5626, 1977.

[6] A. A. Maradudin, E. R. Méndez, and T. A. Leskova, Designer Surfaces, Elsevier Science \& Technology, Oxford, UK, 2008, Sec. 2.2.

[7] R. M. Fitzgerald and A. A. Maradudin, "A reciprocal phaseperturbation theory for rough-surface scattering," Waves in Random Media, vol. 4, pp. 275-296, 1994.

[8] I. Simonsen, "Optics of surface disordered systems: a random walk through rough surface scattering phenomena," The European Physical Journal Special Topics, vol. 181, pp. 1-103, 2010.

[9] A. A. Maradudin, T. Michel, A. R. McGurn, and E. R. Méndez, "Enhanced backscattering of light from a random grating," Annals of Physics, vol. 203, pp. 255-307, 1990.

[10] A. A. Maradudin, E. R. Méndez, and T. A. Leskova, Designer Surfaces, Elsevier Science \& Technology, Oxford, UK, 2008, Sec. 2.9.

[11] V. Freilikher, E. Kanzieper, and A. A. Maradudin, "Coherent scattering enhancement in systems bounded by rough interfaces,” Physics Reports, vol. 288, pp. 127-204, 1997, Appendix A. 


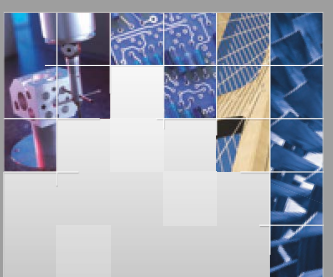

\section{Enfincering}
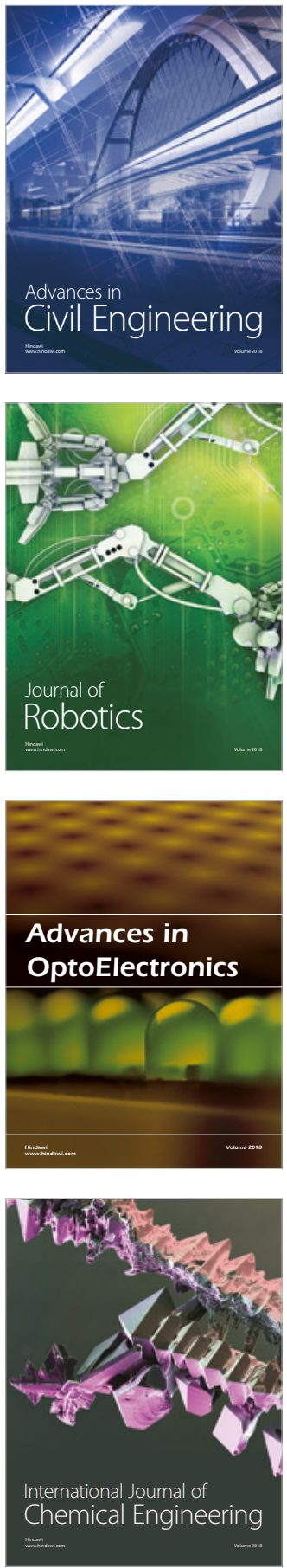

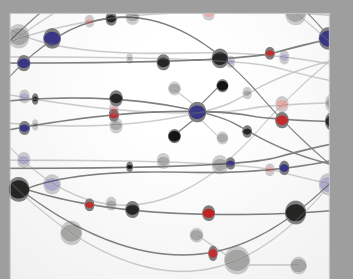

\section{Rotating \\ Machinery}

The Scientific World Journal

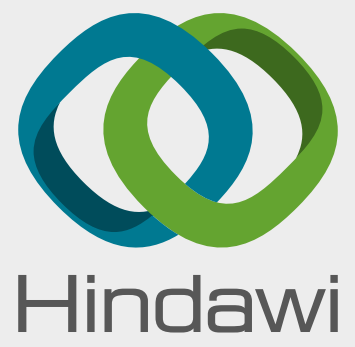

Submit your manuscripts at

www.hindawi.com
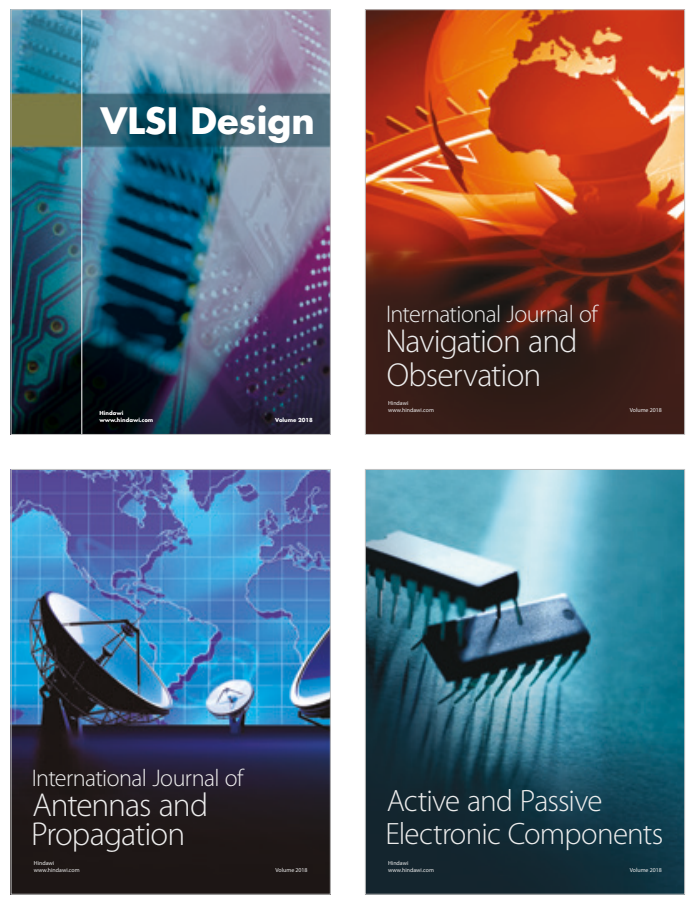
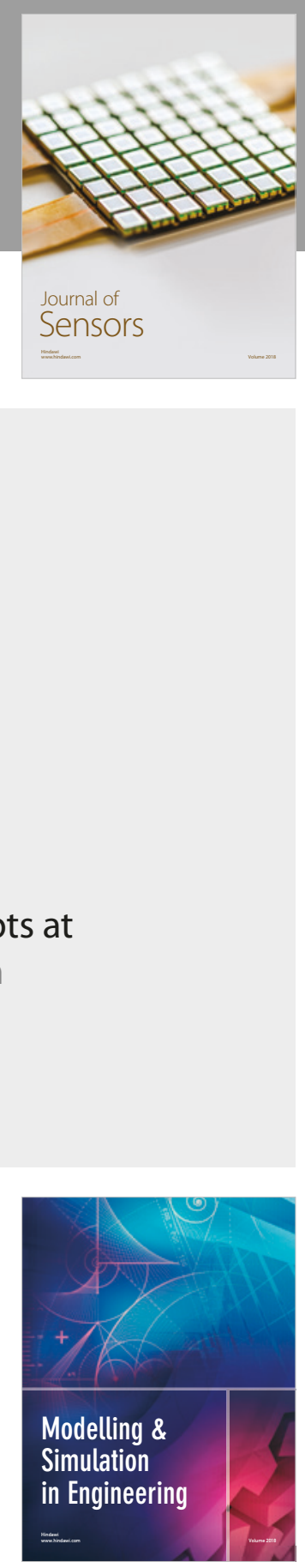

\section{Advances \\ Multimedia}
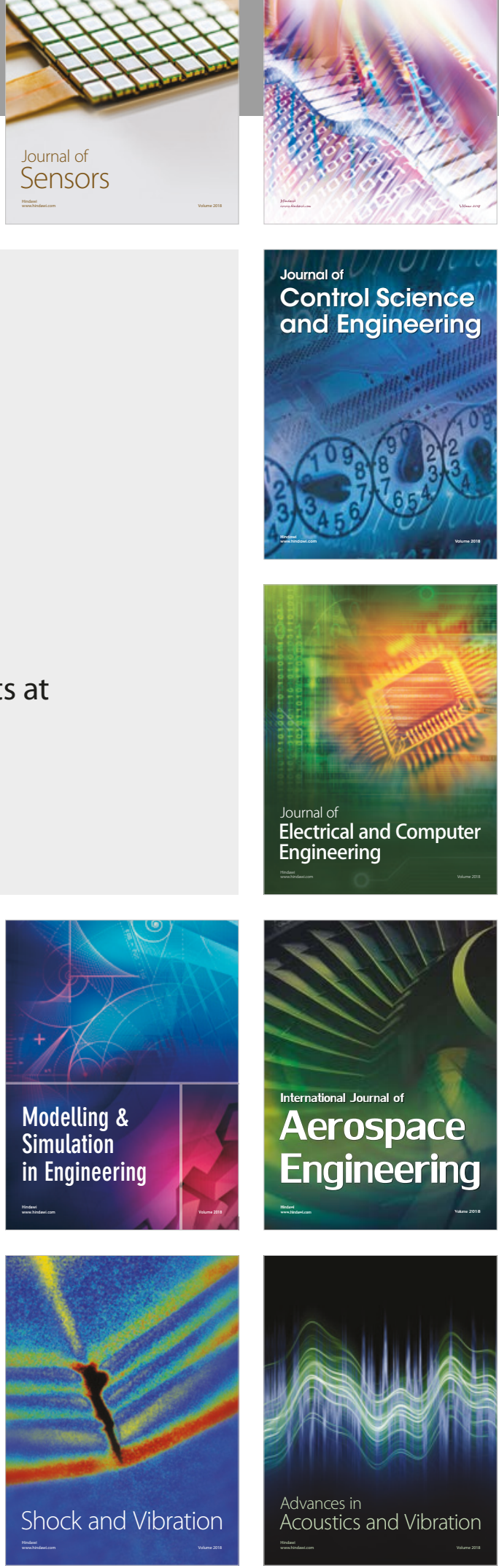